\title{
Estimation of the apparent glass transition temperature of spray- dried emulsified powders and yeast powders by their flavor release behavior under temperature ramping conditions
}

\author{
Sultana, A..$^{\text {a,b,c }}$;hu, J. L..$^{\text {a }}$ Yoshii, H. ${ }^{\text {a,b* }}$ \\ ${ }^{\text {a }}$ Department of Applied Biological Science, Kagawa University, Japan \\ ${ }^{\mathrm{b}}$ Department of Applied Bioresource Science, Ehime University, Japan \\ ${ }^{c}$ Department of Food Processing and Engineering, Chittagong Veterinary and Animal Sciences \\ University, Bangladesh
}

*E-mail of the corresponding author: foodeng.yoshii@ag.kagawa-u.ac.jp

\begin{abstract}
Flavor release from powders depends on the glass transition temperature $\left(T_{g}\right)$ and water absorption. This study reports a simple method to estimate the apparent $T_{g}$ by aroma sensing upon flavor release from spray-dried powders. Four different wall materials (maltodextrin (MD), MD blends with fructose at 20 and $40 w t \%$, and yeast cells) were used to encapsulate flavors by spraydrying. The apparent $T_{g}$ values of MD and MD with 20 and 40wt\% fructose were determined as 130, 110, and $75{ }^{\circ} \mathrm{C}$, respectively, similar to those calculated by the Gordon-Taylor equation. The slow flavor release from driedyeast powder afforded the highest $T_{g}$.
\end{abstract}

Keywords: yeast; flavor; encapsulation; spray drying; aroma sensor. 
Estimation of the apparent glass transition temperature of spray-dried emulsified powders and yeast powders by their flavor release behavior under temperature ramping conditions

\section{Introduction}

Spray drying is a unique technique to encapsulate active compounds. The rapid drying rate, availability of the instrumentation, and continuous operation processing make spray drying a widely useable technique in the encapsulation field. Additionally, good quality powders and high flavor-retention powders can be obtained by spray drying. Encapsulation is the entrapment of an active material inside a wall material. The encapsulation of flavors in solid powders has great benefits as it can reduce the volatility losses, protect from adverse environments, and increase their shelf-life and stability. The encapsulation efficiency differs vastly among different wall and core materials. The glass transition temperature $\left(T_{g}\right)$ is a key property that affects the release of flavors from encapsulated powders.[1] Chirife and Karel[2] and Roos and Karel[3] have described how flavor retention or release from amorphous matrices may be due to the transformation of said amorphous matrices from a glassy to a rubbery phase. They highlighted two causes for this transition: an increase in the moisture content and an elevated temperature. At the $\mathrm{T}_{\mathrm{g}}$, the molecular movement in the powder increases and the amorphous material changes from a glassy to a rubbery state, with consequences of stickiness and collapse of the powder.[4] Furthermore, the stickiness of the powder observed at the $\mathrm{T}_{\mathrm{g}}$ results in poor quality and low yields during drying, and handling and storage problems.[5] Therefore, the determination of the $T_{g}$ of wall materials is very important for application in encapsulating powders. Usually, the glass transition temperature is measured by differential scanning calorimetry (DSC). However, the determination of $\mathrm{T}_{\mathrm{g}}$ values by DSC is very difficult due to the small changes in the heat capacity near the $\mathrm{T}_{\mathrm{g}}$.

In this study, a method for the measurement of the $T_{g}$ of flavor-encapsulated spray-dried emulsified powders and yeast powders is proposed using a simple instrument named aroma sensor. The rapid increment in flavor release from the encapsulating powders was monitored using a ramping method (linear programmed temperature gradient).

\section{Materials and Methods}

\subsection{Materials}

Drum-dried yeast cells, Saccharomyces cerevisiae cells (partially $\beta$-glucan extracted), were obtained from Fuji Foods Corp. (Yokohama, Japan). Maltodextrin (MD, DE = 19) and fructose (Fr) were kindly donated by Matsutani Chemical Industries, Ltd. (Itami, Japan). Sodium caseinate, the emulsifier, was received from Mitsubishi-Chemical Foods Corp. (Tokyo, Japan). The flavors, i.e., the core materials, hexanol, $d$-limonene, and ethyl hexanoate were purchased from Wako Pure Chemical Industries, Ltd. (Osaka, Japan). All 
other chemicals were of analytical grade and obtained from Wako Pure Chemical Industries, Ltd. (Osaka, Japan).

\subsection{Methods}

\subsubsection{Preparation of d-limonene and ethyl hexanoate-encapsulated yeast powders}

$d$-Limonene and ethyl hexanoate were encapsulated in yeast cells following the method described by Sultana et al.[6]

\subsubsection{Preparation of hexanol-encapsulated emulsified powders}

Three different powders were prepared by changing the MD-to-Fr ratio in the feed solution. Powders of MD (100\%) and blends of MD and Fr at ratios of 4:1 and 3:2 were prepared using a pilot spray dryer Ohkawara-L8 (Ohkawara Kakouki Co., Ltd., Yokohama, Japan) equipped with a centrifugal atomizer. Feed solutions were prepared by dissolution of MD or its blends with Fr (38.5 wt $\%)$ together with sodium caseinate (1.5 wt $\%)$ and hexanol in medium chain triglyceride (MCT) oil $(10 \mathrm{wt} \%)$ in distilled water (50 wt $\%)$. The solid content was maintained at $50 \mathrm{wt} \%$. Hexanol was diluted in MCT oil at a ratio of 1:4 (\%wt). The mixture was homogenized using a mechanical homogenizer, a polytron homogenizer (PT-10, Kinematica GA, Littau, Switzerland), at $8000 \mathrm{rpm}$ for $3 \mathrm{~min}$. Finally, the carrier solution was spray-dried at an inlet air temperature of $160{ }^{\circ} \mathrm{C}$, atomizer speed of $10,000 \mathrm{rpm}$, and feed flow of $20 \mathrm{~mL} / \mathrm{min}$. The outlet temperature was monitored at $83-106{ }^{\circ} \mathrm{C}$.

\subsubsection{Analysis of the d-limonene and ethyl hexanoate extracted from yeast powder}

The encapsulated $d$-limonene and ethyl hexanoate were extracted from yeast cells and analyzed by gas chromatography following the procedure described by Sultana et al.[7]

\subsubsection{Analysis of hexanol from flavor-encapsulated powders}

The flavor-encapsulated powder $(0.1 \mathrm{~g})$ was completely dissolved in $5 \mathrm{~mL}$ of distilled water in a glass bottle. Diethyl ether with cyclohexanone (5 mL of $1000 \mathrm{ppm}$ ) was added as the internal standard and the mixture was vortexed for $20 \mathrm{~min}$ at $20{ }^{\circ} \mathrm{C}$ in a high speed shaker (CM-1000, Tokyo Rikakikai Co., Ltd., Tokyo, Japan). After centrifugation, a small amount of anhydrous sodium sulfate was added to the upper phase and $1 \mu \mathrm{L}$ was injected in a gas chromatograph-mass spectrometer (GC-MS, Shimadzu Corp., Kyoto, Japan). The GC-MS 
Estimation of the apparent glass transition temperature of spray-dried emulsified powders and yeast powders by their flavor release behavior under temperature ramping conditions

was equipped with a fused silica capillary column $(30 \mathrm{~m} \times 0.25 \mathrm{~mm} \times 0.25 \mu \mathrm{m}$; DB-1, Agilent Technologies Japan Ltd., Tokyo, Japan). The column temperature was programmed from 80 to $260{ }^{\circ} \mathrm{C}$ at a rate of $3{ }^{\circ} \mathrm{C} / \mathrm{min}$ and from 260 to $300{ }^{\circ} \mathrm{C}$ at $20{ }^{\circ} \mathrm{C} / \mathrm{min}$, where the temperature was maintained constant for $28 \mathrm{~min}$.

\subsubsection{Measurement of the apparent $T_{g}$ of flavor-encapsulated powders with aroma sensor}

The flavor-encapsulated powder $(1 \mathrm{~g})$ was placed in a glass vessel $\left(24^{\mathrm{d}} \times 45^{\mathrm{h}} \mathrm{mm}\right)$ and covered with a rubber cap. The cap of the glass vessel had holes for two needles, one for nitrogen supply and the other one for air sampling. The air sampling port was connected to a handheld odor meter (KANOMAX, OMX-SRM model, Kobe, Japan) through a polyurethane tube (PISCO, Okayama, Japan). The released flavors were detected by the aroma sensor. A nitrogen flow rate of 30-50 $\mathrm{mL} / \mathrm{min}$ was maintained depending on the aroma strength of the sample. To determine the aroma strength of the encapsulated yeast powder, a Tee connector was connected to the tube from the sampling vessel to the sensor to dilute the flavor concentration. The vessel was placed in an aluminum block on a digital plate heater, which was used to control the temperature of the powder at two different ramp rates $(0.5$ and $\left.1^{\circ} \mathrm{C} / \mathrm{min}\right)$. The powder temperature was recorded with a data logger. The data filing software OMX-SRM was installed in the computer to collect the released flavor data during the whole process. The apparent $T_{g}$ was the intercept temperature of the tangent line in the flavor release plot.

\section{Results and Discussion}

\subsection{Measurement of the apparent $T_{g}$ of emulsified flavor powders}

The moisture content of the emulsified flavor powders was below $2 \%$, whereas the retention of encapsulated hexanol in the MD (DE = 19) and MD with 20 or $40 \mathrm{wt} \%$ Fr samples was about $100 \%, 70 \%$, and $65 \%$, respectively. Figure 1 shows the hexanol release behavior of the emulsified powders with the increasing temperature at rates of 1 and $0.5{ }^{\circ} \mathrm{C} / \mathrm{min}$. The flavor strength gradually increased with the temperature, even though the flavor strength was very low near $20^{\circ} \mathrm{C}$. However, the strength of the flavor sharply increased at a certain temperature, indicating the glass transition temperature of the wall material. The apparent glass transition temperature is the intercept point of the tangent line for the hexanol release rate (flavor strength detected by the aroma sensor). The apparent glass transition temperature of the MD (DE = 19) and MD with 20 and $40 \mathrm{wt} \%$ Fr containing powders were determined to be around 130,110 , and $75^{\circ} \mathrm{C}$, respectively. 

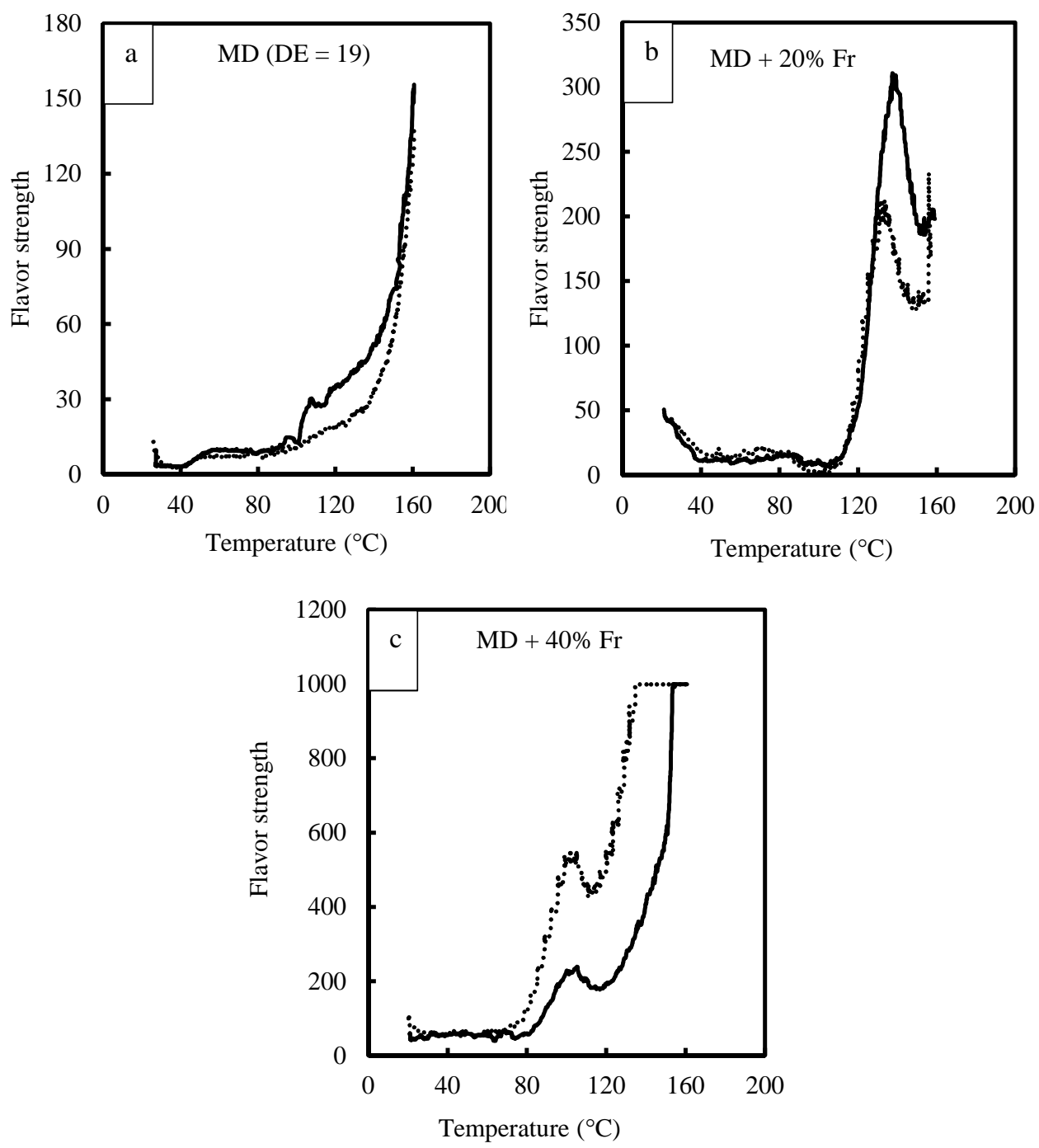

Fig. 1 Hexanol release behavior of emulsified powders: (a) maltodextrin (DE =19), (b) MD with $20 \mathrm{wt} \% \mathrm{Fr}$, and (c) $\mathrm{MD}$ with $40 \mathrm{wt} \% \mathrm{Fr}$ (solid lines: $1^{\circ} \mathrm{C} / \mathrm{min}$; dotted lines: $0.5^{\circ} \mathrm{C} / \mathrm{min}$ ).

It can be seen that the addition of Fr to MD reduces its glass transition temperature. The low $\mathrm{T}_{\mathrm{g}}$ of pure $\mathrm{Fr}$, which was reported by Roos[8] to be $5{ }^{\circ} \mathrm{C}$, reduces the apparent $\mathrm{T}_{\mathrm{g}}$ of the wall material. It must be noted that the $\mathrm{T}_{\mathrm{g}}$ values for the above mentioned powders calculated using the Gordon-Taylor equation are very similar to the experimental values shown in the graphs. 


\subsection{Measurement of the apparent $T_{g}$ of flavor-encapsulated yeast powders}
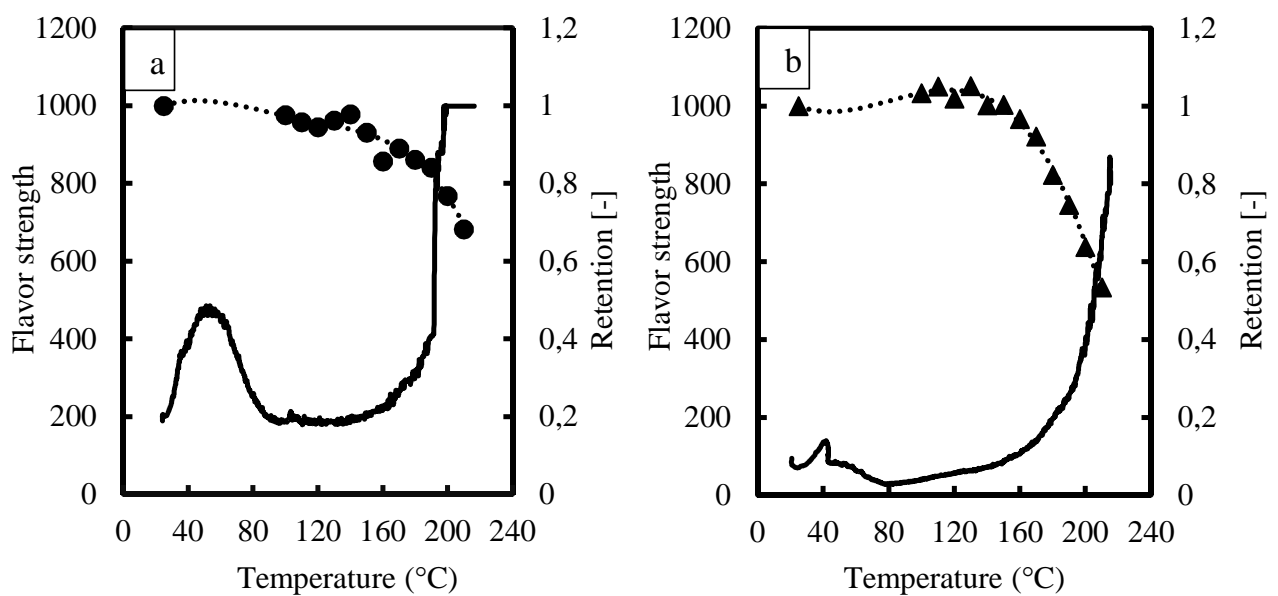

Fig. 2 Release behavior of (a) ethyl hexanoate and (b) d-limonene from yeast powders (solid lines: flavor strength; dotted lines: retention of flavor).

Figure 2 illustrates the release behavior of ethyl hexanoate and $d$-limonene from yeast cells with the increasing temperature at $1{ }^{\circ} \mathrm{C} / \mathrm{min}$. The initial high flavor strength possibly corresponds to the surface flavor of the yeast powder. After that, the flavor strength decreases to finally increase again at very high temperatures $\left(180-190{ }^{\circ} \mathrm{C}\right)$. The complex structure of yeast, i.e., the presence of different sugars, proteins, and others species, hampers the accurate monitoring of the flavor release. To confirm the apparent $T_{g}$ of the flavor-encapsulated yeast powders, $d$-limonene and ethyl hexanoate were extracted from yeast cells under ramping temperature conditions (at $1{ }^{\circ} \mathrm{C} / \mathrm{min}$ ) and analyzed by GC-FID. The retention of the encapsulated flavor was calculated following the method by Sultana et al.[6] The obtained intercept point was very similar to the apparent $T_{g}$ value found using the aroma sensor. The presence of trehalose, sucrose, and other sugars in yeast cells may be the reason behind the high apparent $\mathrm{T}_{\mathrm{g}}$ of yeast cells.

\section{Conclusions}

The apparent glass transition temperatures of flavor encapsulated spray-dried powders were successfully determined using an aroma sensor. The apparent $\mathrm{T}_{\mathrm{g}}$ of powders composed of MD (DE = 19) and MD with 20 and $40 \mathrm{wt} \%$ Fr were approximately 130, 110, and $75^{\circ} \mathrm{C}$, respectively. The increasing temperature rate did not significantly affect the $T_{g}$ of the encapsulating powders. In contrast, yeast cells exhibited a slower release of flavors and higher $\mathrm{T}_{\mathrm{g}}$ value. 


\section{Acknowledgement}

Afroza Sultana would like to thank the Japan Ministry of Education, Culture, Sports, Science and Technology for providing a scholarship (Monbukagakusho: MEXT).

\section{References}

[1] Carolina, B.C.; Carolina, S.; Zamora, M.C.; Jorge, C. Glass transition temperatures and some physical and sensory changes in stored spray-dried encapsulated flavors. LWT Food Science and Technology 2007, 40, 1792-1797.

[2] Chirife, J.; Karel, M. Effect of structure disrupting treatments on volatile release from freeze-dried maltose. Journal of Food Technology 1974, 9, 13-20.

[3] Roos, Y.; Karel, M. Plasticizing effect of water on thermal behavior and crystallization of amorphous food models. Journal of Food Science 1991, 56, 38-43.

[4] Levine, H.; Slade, L. A polymer physico-chemical approach to the study of commercial starch hydrolysis products (SHPs). Carbohydrate Polymers 1986, 6, 213-244.

[5] Shrestha, A.K.; Howes, T.; Adhikari, B.P.; Wood, B.J.; Bhandari, B.R. Effect of protein concentration on the surface composition, water sorption and glass transition temperature of spray-dried skim milk powders. Food Chemistry 2007, 104, 1436-1444.

[6] Sultana, A.; Tanaka, Y.; Fushimi, Y.; Yoshii, H. Stability and release behavior of encapsulated flavor from spray-dried Saccharomyces cerevisiae and maltodextrin powder. Food Research International 2018, 106, 809-816.

[7] Sultana. A.; Miyamoto, A.; Hy, Q.L.; Tanaka, Y.; Fushimi, Y.; Yoshii, H. Microencapsulation of flavors by spray drying using Saccharomyces cerevisiae. Journal of Food Engineering 2017, 199, 36-41.

[8] Roos, Y. Characterization of food polymers using state diagrams. Journal of Food Engineering 1995, 24, 339-360. 